PROCEEDINGS OF THE

AMERICAN MATHEMATICAL SOCIETY

Volume 131, Number 6, Pages 1867-1874

S 0002-9939(02)06786-2

Article electronically published on November 6, 2002

\title{
LOCAL AUTOMORPHISMS OF OPERATOR ALGEBRAS ON BANACH SPACES
}

\author{
LAJOS MOLNÁR
}

(Communicated by David R. Larson)

\begin{abstract}
In this paper we extend a result of Šemrl stating that every 2-local automorphism of the full operator algebra on a separable infinite dimensional Hilbert space is an automorphism. In fact, besides separable Hilbert spaces, we obtain the same conclusion for the much larger class of Banach spaces with Schauder bases. The proof rests on an analogous statement concerning the 2-local automorphisms of matrix algebras for which we present a short proof. The need to get such a proof was formulated in Šemrl's paper.
\end{abstract}

\section{INTRODUCTION AND STATEMENT OF THE RESULTS}

The importance of the study of automorphism groups of algebraic structures needs no justification. Recently, we have published several results on the local behaviour of the automorphism groups of operator algebras. Roughly speaking, we have studied the question of how the automorphisms of certain operator algebras (or of function algebras) are determined by their local actions. One area of such investigations was initiated by P. Šemrl who introduced the concept of 2local automorphisms. If $\mathcal{A}$ is any algebra, then a transformation (no linearity is assumed) $\phi: \mathcal{A} \rightarrow \mathcal{A}$ is called a 2-local automorphism of $\mathcal{A}$ if for every $A, B \in \mathcal{A}$ there exists an (algebra) automorphism $\phi_{A, B}$ of $\mathcal{A}$ such that $\phi(A)=\phi_{A, B}(A)$ and $\phi(B)=\phi_{A, B}(B)$. It is then a remarkable fact on the algebra $\mathcal{A}$ if every 2-local automorphism of $\mathcal{A}$ is an automorphism. Observe that for 1-local automorphisms (the definition should be self-explanatory) without any extra properties, reasonable results of that kind cannot be expected.

The first result on 2-local automorphisms is due to Šemrl [9] who proved that if $H$ is a separable infinite dimensional Hilbert space, then every 2-local automorphism of the algebra $B(H)$ of all bounded linear operators on $H$ is an automorphism. Moreover, since in the finite dimensional case he was only able to get a long proof involving tedious computations, Šemrl thought that it would be of some interest to produce a short proof in that case. One aim of this paper is to present such a proof. Furthermore, we extend Šemrl's result quite significantly, namely, in addition to

Received by the editors November 22, 2000 and, in revised form, February 6, 2002.

2000 Mathematics Subject Classification. Primary 47B49, 16 S50.

Key words and phrases. Automorphism, local automorphism, matrix algebra, operator algebra. This research was supported by the Hungarian National Foundation for Scientific Research (OTKA), Grant No. T030082, T031995, and by the Ministry of Education, Hungary, Reg. No. FKFP 0349/2000. 
the case of separable Hilbert spaces, we get the same conclusion for general Banach spaces with Schauder bases. We recall that most classical separable Banach spaces have Schauder bases (see the first chapter in [2]) and hence our generalization really has sense.

Now we turn to the formulation of our assertions. If $\mathbb{F}$ is a field, then $M_{n}(\mathbb{F})$ stands for the algebra of all $n \times n$ matrices with entries in $\mathbb{F}$. It is well-known that the automorphisms of the algebra $M_{n}(\mathbb{F})$ are exactly the transformations $A \mapsto T A T^{-1}$ where $T \in M_{n}(\mathbb{F})$ is nonsingular (see, for example, 7, Lemma, p. 230]). In what follows, if $A \in M_{n}(\mathbb{F})$, we denote by $\operatorname{eigv}(A)$ the system of all eigenvalues of $A$ listed according to multiplicity. We emphasize that eigv $(A)$ generally differs from the spectrum of $A$ as a linear operator.

Our first theorem gives a nonlinear characterization of the automorphisms of $M_{n}(\mathbb{F})$.

Theorem 1. Suppose that $\mathbb{F}$ is an algebraically closed field of characteristic 0 . Let $\phi: M_{n}(\mathbb{F}) \rightarrow M_{n}(\mathbb{F})$ be a transformation (linearity is not assumed) such that

$$
\operatorname{eigv}(\phi(A) \phi(B))=\operatorname{eigv}(A B)
$$

holds for every $A, B \in M_{n}(\mathbb{F})$. Then there exists a nonsingular matrix $T \in M_{n}(\mathbb{F})$ and $\lambda \in\{-1,1\}$ such that $\phi$ is either of the form

$$
\phi(A)=\lambda T A T^{-1} \quad\left(A \in M_{n}(\mathbb{F})\right)
$$

or of the form

$$
\phi(A)=\lambda T A^{t} T^{-1} \quad\left(A \in M_{n}(\mathbb{F})\right) .
$$

We mention that similar characterizations of the automorphisms of operator algebras and of function algebras can be found in [4], but there we had to assume that the transformations in question are all surjective. The main advantage of the present theorem is that we can omit that assumption, and this fact makes it possible to obtain the following corollary concerning 2-local automorphisms of matrix algebras over general fields.

Corollary 2. Let $\mathbb{F}$ be an algebraically closed field of characteristic 0 . Then every 2-local automorphism of $M_{n}(\mathbb{F})$ is an automorphism.

Since the field $\mathbb{R}$ of real numbers is not algebraically closed, we formulate the analogous statement for $M_{n}(\mathbb{R})$ as a separate proposition.

Proposition 3. Every 2-local automorphism of $M_{n}(\mathbb{R})$ is an automorphism.

The arguments applied in the proof of our results above lead us to an extension of Šemrl's theorem for the case of certain subalgebras of the algebra $B(X)$ of all bounded linear operators on the Banach space $X$ with a Schauder basis. We believe that with some extra work Šemrl's original proof could also be improved to produce the following result (although only in the infinite dimensional case), but our proof has the advantage that it puts both the finite and the infinite dimensional cases into the same perspective.

Theorem 4. Let $X$ be a real or complex Banach space with a Schauder basis. Suppose that $\mathcal{A}$ is a subalgebra of $B(X)$ which contains the ideal of all compact operators on $X$. If $\phi$ is a 2-local automorphism of $\mathcal{A}$, then $\phi$ is an automorphism of $\mathcal{A}$. 


\section{Proofs}

In the proof of our first result we shall need the following folklore result whose proof requires only elementary linear algebra. If $X$ is a linear space over $\mathbb{F}$ and $A, B: X \rightarrow X$ are linear operators of rank at least 2 with the property that for every $x \in X$ the vectors $A x, B x$ are linearly dependent, then the operators $A, B$ are linearly dependent.

Proof of Theorem 1. Since the statement is obvious for $n=1$, we suppose that $n \geq 2$. Denote by tr the usual trace functional on $M_{n}(\mathbb{F})$. It follows from the property (1), i.e., from the equality $\operatorname{eigv}(\phi(A) \phi(B))=\operatorname{eigv}(A B)$ that

$$
\operatorname{tr} \phi(A) \phi(B)=\operatorname{tr} A B
$$

holds for every $A, B \in M_{n}(\mathbb{F})$.

As usual, denote by $E_{i j} \in M_{n}(\mathbb{F})$ the matrix whose $i j$ entry is 1 and all its other entries are 0 . We assert that the $\phi\left(E_{i j}\right)$ 's are linearly independent. Suppose that

$$
\sum_{i, j} \lambda_{i j} \phi\left(E_{i j}\right)=0
$$

for some scalars $\lambda_{i j} \in \mathbb{F}$. Fix indices $k, l \in\{1, \ldots, n\}$. We have

$$
\sum_{i, j} \lambda_{i j} \phi\left(E_{i j}\right) \phi\left(E_{k l}\right)=0
$$

Taking trace, we obtain

$$
\sum_{i, j} \lambda_{i j} \operatorname{tr} \phi\left(E_{i j}\right) \phi\left(E_{k l}\right)=0
$$

By the property (2) of $\phi$, it follows that

$$
\sum_{i, j} \lambda_{i j} \operatorname{tr} E_{i j} E_{k l}=0 .
$$

Since $E_{i j} E_{k l}=\delta_{j k} E_{i l}$, from this equality we easily deduce that $\lambda_{l k}=0$. As $k, l$ are arbitrary, it follows that the matrices $\phi\left(E_{i j}\right), i, j \in\{1, \ldots, n\}$, form a linearly independent set in $M_{n}(\mathbb{F})$. This implies that the range of $\phi$ linearly generates $M_{n}(\mathbb{F})$.

We are now in a position to prove that $\phi$ is linear. If $A, B \in M_{n}(\mathbb{F})$, we compute

$$
\begin{gathered}
\operatorname{tr} \phi(A+B) \phi(C)=\operatorname{tr}(A+B) C=\operatorname{tr} A C+\operatorname{tr} B C \\
=\operatorname{tr}(\phi(A) \phi(C)+\phi(B) \phi(C))=\operatorname{tr}(\phi(A)+\phi(B)) \phi(C)
\end{gathered}
$$

for any $C \in M_{n}(\mathbb{F})$. Since the linear span of the $\phi(C)$ 's is the whole space $M_{n}(\mathbb{F})$, it follows that $\phi(A+B)=\phi(A)+\phi(B)$. The homogenity of $\phi$ can be proved in a very similar way. So, $\phi$ is a surjective linear transformation on $M_{n}(\mathbb{F})$ which is hence bijective.

Let $A \in M_{n}(\mathbb{F})$ be of rank one. Then, using (1) and the surjectivity of $\phi$, it follows that $\phi(A) B, B \phi(A)$ both have at most one nonzero eigenvalue and its multiplicity is one. This implies that $\phi(A)$ is of rank one. Consequently, $\phi$ preserves the rank-one elements of $M_{n}(\mathbb{F})$. The form of all such linear transformations on $M_{n}(\mathbb{F})$ is well-known. It follows from [3] that there are nonsingular matrices $T, S$ in $M_{n}(\mathbb{F})$ such that $\phi$ is either of the form

$$
\phi(A)=T A S \quad\left(A \in M_{n}(\mathbb{F})\right)
$$


or of the form

$$
\phi(A)=T A^{t} S \quad\left(A \in M_{n}(\mathbb{F})\right) .
$$

Without loss of generality we can assume that $\phi$ is of the first form. Composing our transformation $\phi$ with the automorphism $A \mapsto S A S^{-1}$, it follows that we can assume that the matrix $S$ appearing in (3) is the identity $I$. We have

$$
\operatorname{tr} T A T B=\operatorname{tr} A B
$$

for every $A, B \in M_{n}(\mathbb{F})$. If we fix $A \in M_{n}(\mathbb{F})$ for a moment and let $B$ run through $M_{n}(\mathbb{F})$, it follows from (44) that $T A T=A$. Now, if $A$ runs through the set of all rank-one matrices in $M_{n}(\mathbb{F})$, we deduce from the equality $T A T=A$ that $T$, as a linear operator on the linear space $X$, has the property that for every $x \in X$, the vector $T x$ is a scalar multiple of $x$. This gives us that $T$ is of the form $\lambda I$ for some scalar $\lambda \in \mathbb{F}$. Clearly, we have $\lambda^{2}=1$ and this completes the proof of our statement.

Proof of Corollary 2 First we again recall that the automorphisms of $M_{n}(\mathbb{F})$ are exactly the transformations of the form $A \mapsto T A T^{-1}$ with some nonsingular matrix $T \in M_{n}(\mathbb{F})$. Now let $\phi: M_{n}(\mathbb{F}) \rightarrow M_{n}(\mathbb{F})$ be a 2-local automorphism. Clearly, $\phi$ satisfies (11). Since $\phi(I)=I$, we infer from Theorem 1 that there exists a nonsingular matrix $T \in M_{n}(\mathbb{F})$ such that $\phi$ is either of the form

$$
\phi(A)=T A T^{-1} \quad\left(A \in M_{n}(\mathbb{F})\right)
$$

or of the form

$$
\phi(A)=T A^{t} T^{-1} \quad\left(A \in M_{n}(\mathbb{F})\right) .
$$

Choosing $A, B \in M_{n}(\mathbb{F})$ such that $A B=0, B A \neq 0$, it follows from the 2-local property of $\phi$ that this second possibility above is excluded and we obtain the desired conclusion.

Proof of Proposition 3. The statement follows from obvious modifications of the proof of Corollary 2 and that of Theorem [1. The only difference is that instead of referring to the result of Marcus and Moyls on the form of all linear transformations on $M_{n}(\mathbb{F})$ preserving rank-one matrices, here we have to refer to an analogous statement concerning the algebra of matrices over the real field due to Omladič and Šemrl [5, Main Theorem].

In the proof of Theorem 4 we need the following notation and definitions. Let $X$ be a (real or complex) Banach space. The algebra of all bounded linear operators on $X$ is denoted by $B(X)$ and $F(X)$ stands for the ideal of all finite rank operators in $B(X)$. An operator $P \in B(X)$ is called an idempotent if $P^{2}=P$. Two idempotents $P, Q$ in $B(X)$ are said to be (algebraically) orthogonal if $P Q=Q P=0$. The dual space of $X$ (that is, the set of all bounded linear functionals on $X$ ) is denoted by $X^{\prime}$. The Banach space adjoint of an operator $A \in B(X)$ is denoted by $A^{\prime}$. If $x \in X$ and $f \in X^{\prime}$, then $x \otimes f$ stands for the operator (of rank at most 1 ) defined by

$$
(x \otimes f)(z)=f(z) x \quad(z \in X) .
$$

It requires only elementary computation to show that

$$
A \cdot x \otimes f=(A x) \otimes f, \quad x \otimes f \cdot A=x \otimes\left(A^{\prime} f\right), \quad x \otimes f \cdot y \otimes g=f(y) x \otimes g
$$


hold for every $x, y \in X, f, g \in X^{\prime}$ and $A \in B(X)$. It is easy to see that the elements of $F(X)$ are exactly the operators $A \in B(X)$ which can be written as a finite sum

$$
A=\sum_{i} x_{i} \otimes f_{i}
$$

for some $x_{1}, \ldots, x_{n} \in X$ and $f_{1}, \ldots, f_{n} \in X^{\prime}$. Using this representation, the trace of $A$ is defined by

$$
\operatorname{tr} A=\sum_{i} f_{i}\left(x_{i}\right) .
$$

It is known that $\operatorname{tr} A$ is well-defined, that is, it does not depend on the particular representation (5) of $A$. It is easy to see that tr is a linear functional on $F(X)$ with the property that

$$
\operatorname{tr} T A=\operatorname{tr} A T
$$

holds for every $A \in F(X)$ and $T \in B(X)$. Finally, we recall that, similar to the case of matrix algebras, every automorphism of any subalgebra of $B(X)$ which contains $F(X)$ (these are the so-called standard operator algebras on $X$ ) is of the form $A \mapsto T A T^{-1}$ where $T \in B(X)$ is invertible (see, for example, [8]).

Proof of Theorem 4. In view of our previous results we can assume that $X$ is infinite dimensional. Let $\phi$ be a 2-local automorphism of $\mathcal{A}$. If $P \in \mathcal{A}$ is a finite rank idempotent, then $\phi(P)=\tilde{P}$ is also a finite rank idempotent and the ranks of $P$ and $\tilde{P}$ are the same. Consider the subalgebra $\mathcal{A}_{P}$ of $\mathcal{A}$ which consists of all operators $A \in \mathcal{A}$ for which $P A P=A$. It follows from the 2-local property of $\phi$ that $\phi(P) \phi(A) \phi(P)=\phi(A)$. Consequently, $\phi$ maps $\mathcal{A}_{P}$ into $\mathcal{A}_{\tilde{P}}$. Clearly, both algebras $\mathcal{A}_{P}$ and $\mathcal{A}_{\tilde{P}}$ are isomorphic to $M_{n}(\mathbb{K})$ (K⿺ stands for the real or complex field) where $n=\operatorname{rank} P=\operatorname{rank} \tilde{P}$. It is easy to see that $\phi$ has the property (2). Now, one can follow the arguments given in the proofs of our previous results to show that $\phi$ is linear and multiplicative on $\mathcal{A}_{P}$. Since $P \in \mathcal{A}$ was an arbitrary finite rank idempotent, we can infer that the restriction $\psi$ of $\phi$ onto $F(X)$ is an algebra endomorphism of $F(X)$ which preserves the rank.

Since $\psi$ is an algebra homomorphism of $F(X)$, we have an injective linear operator $T: X \rightarrow X$ such that

$$
T A=\psi(A) T \quad(A \in F(X)) .
$$

Indeed, similarly as in [8] we define

$$
T x=\psi\left(x \otimes f_{0}\right) y_{0} \quad(x \in X)
$$

where $y_{0} \in X$ and $f_{0} \in X^{\prime}$ are fixed such that $\psi\left(x \otimes f_{0}\right) y_{0} \neq 0$ for some $x \in X$. It is evident that $T$ is a nonzero linear operator on $X$. It follows from the multiplicativity of $\psi$ that $T A=\psi(A) T(A \in F(X))$. To see the injectivity of $T$, let $T x=0$ and $x \neq 0$. Then we have $T A x=\psi(A) T x=0$ for every $A \in F(X)$. This gives us that $T y=0$ holds for every $y \in X$ which is an obvious contradiction.

We show that $T$ is continuous. To see this, we apply the closed graph theorem. Let $\left(x_{n}\right)$ be a sequence in $X$ and $y \in X$ be such that $x_{n} \rightarrow 0$ and $T x_{n} \rightarrow y$. We have to show that $y=0$. Since $T A$ is continuous for every $A \in F(X)$, from (6) we infer that

$$
\psi(A) y=0 \quad(A \in F(X)) .
$$


Here is the point where we have to use that $X$ has a Schauder basis. Namely, this condition implies that we have a sequence $\left(P_{n}\right)$ of pairwise orthogonal rankone idempotents in $B(X)$ whose sum strongly converges to $I$. In particular, $\left(P_{n}\right)$ is uniformly bounded. Clearly, we can write $P_{n}=x_{n} \otimes f_{n}$ where $x_{n} \in X, f_{n} \in X^{\prime}$ are such that $f_{i}\left(x_{j}\right)=\delta_{i j}$ (the Kronecker symbol) and $\left\|x_{i}\right\|<m,\left\|f_{j}\right\|<M$ $(i, j \in \mathbb{N})$ for some positive real numbers $m, M$. Choose a sequence $\left(\lambda_{n}\right)$ of positive real numbers with the property that $\sum_{n=k+1}^{\infty} \lambda_{n}<\lambda_{k}$ holds for every $k \in \mathbb{N}$. For example, one can define $\lambda_{n}=(1 / 3)^{n}(n \in \mathbb{N})$. In particular, it follows that $\sum_{n} \lambda_{n} P_{n}$ converges in the norm topology and hence its sum is a compact operator. Pick another sequence $\left(\mu_{n}\right)$ of positive real numbers for which $\sum_{n} \mu_{n}<\infty$. The operator $\sum_{n} \mu_{n} x_{n} \otimes f_{n+1}$ is also compact.

By the 2-local property of $\phi$, composing $\phi$ with an automorphism of $\mathcal{A}$ if necessary, we can (and do) assume that for the particular operators $\sum_{n} \lambda_{n} P_{n}$ and $\sum_{n} \mu_{n} x_{n} \otimes f_{n+1}$ we have

$$
\phi\left(\sum_{n} \lambda_{n} P_{n}\right)=\sum_{n} \lambda_{n} P_{n} \quad \text { and } \quad \phi\left(\sum_{n} \mu_{n} x_{n} \otimes f_{n+1}\right)=\sum_{n} \mu_{n} x_{n} \otimes f_{n+1} .
$$

Let $n_{0} \in \mathbb{N}$ be arbitrary. By the 2-local property of $\phi$ we have an invertible bounded linear operator $U \in B(X)$ such that

$$
\phi\left(\sum_{n} \lambda_{n} P_{n}\right)=U\left(\sum_{n} \lambda_{n} P_{n}\right) U^{-1} \quad \text { and } \quad \phi\left(P_{n_{0}}\right)=U P_{n_{0}} U^{-1} .
$$

From

$$
\sum_{n} \lambda_{n} U P_{n} U^{-1}=U\left(\sum_{n} \lambda_{n} P_{n}\right) U^{-1}=\phi\left(\sum_{n} \lambda_{n} P_{n}\right)=\sum_{n} \lambda_{n} P_{n}
$$

we can infer that $U P_{n} U^{-1}=P_{n}$ holds for every $n \in \mathbb{N}$. Indeed, let $U P_{n} U^{-1}=Q_{n}$ $(n \in \mathbb{N})$. Dividing both sides of the equality

$$
\sum_{n} \lambda_{n} Q_{n}=\sum_{n} \lambda_{n} P_{n}
$$

by $\lambda_{1}$ and then taking the $k$ th powers of the operators on both sides and, finally, letting $k$ tend to infinity, by the property of the sequence $\left(\lambda_{n}\right)$ we easily obtain that $Q_{1}=P_{1}$. Therefore, we have

$$
\sum_{n=2}^{\infty} \lambda_{n} Q_{n}=\sum_{n=2}^{\infty} \lambda_{n} P_{n}
$$

and one can proceed in the same way to show that $Q_{n}=P_{n}$ holds for every $n \in \mathbb{N}$. In particular, we have $\phi\left(P_{n_{0}}\right)=Q_{n_{0}}=P_{n_{0}}$. But $n_{0}$ was arbitrary and hence we obtain that

$$
\phi\left(P_{n}\right)=P_{n} \quad(n \in \mathbb{N}) .
$$

We now go back to the proof that $T$ is continuous. By (7) we have $\psi\left(P_{n}\right) y=0$ $(n \in \mathbb{N})$ and this, together with (9), yields that $P_{n} y=0$ holds for every $n \in \mathbb{N}$. This implies that $y=0$ verifying the continuity of $T$.

Besides the fact that $\psi$ is an algebra endomorphism of $F(X)$, we know that $\psi$ is rank preserving. The form of linear rank preservers on operator algebras is known. In fact, a description of such transformations is given, for example, in [1]. It follows from the arguments used in the first half of Section I in [1] that we have 
two possibilities: either there are linear operators $S: X \rightarrow X$ and $R: X^{\prime} \rightarrow X^{\prime}$ such that

$$
\psi(x \otimes f)=S x \otimes R f \quad\left(x \in X, f \in X^{\prime}\right)
$$

or there are linear operators $R: X \rightarrow X^{\prime}$ and $S: X^{\prime} \rightarrow X$ such that

$$
\psi(x \otimes f)=S f \otimes R x \quad\left(x \in X, f \in X^{\prime}\right) .
$$

(To be honest we note that in [1] only complex spaces were considered, but the arguments given there also apply to the real case.) This second possibility can be excluded easily after referring to the multiplicativity of $\psi$. So, we have linear operators $S: X \rightarrow X$ and $R: X^{\prime} \rightarrow X^{\prime}$ such that

$$
\psi(x \otimes f)=S x \otimes R f \quad\left(x \in X, f \in X^{\prime}\right) .
$$

It follows from (6) that

$$
T x \otimes f=T \cdot x \otimes f=S x \otimes R f \cdot T=S x \otimes T^{\prime} R f .
$$

This implies that $T x, S x$ are linearly dependent for every $x \in X$. Hence, $S$ is a scalar multiple of $T$. Therefore, in what follows we can (and do) assume that the linear operator $S$ appearing in (10) is equal to $T$.

By the local form of $\phi$ it follows that $\psi$ is trace preserving. Consequently, we have

$$
(R f)(T x)=f(x) \quad\left(x \in X, f \in X^{\prime}\right) .
$$

This means that $T^{\prime} R$ equals the identity on $X^{\prime}$. In particular, the range of $T^{\prime}$ is closed which is well-known to imply that the range of $T$ is also closed. On the other hand, $T$ has dense range which follows from (6) and (9). Therefore, we can infer that $T, T^{\prime}$ are invertible and $R=T^{\prime-1}=T^{-1^{\prime}}$. This gives us that

$$
\psi(x \otimes f)=T \cdot x \otimes f \cdot T^{-1} \quad\left(x \in X, f \in X^{\prime}\right)
$$

and hence we have $\phi(A)=\psi(A)=T A T^{-1}$ for every $A \in F(X)$. We show that $\phi(A)=T A T^{-1}$ holds also for every $A \in \mathcal{A}$. In order to do this, let $A \in \mathcal{A}$ be arbitrary. Pick any $x \in X, f \in X^{\prime}$ and let $B=x \otimes f$. By the 2-local property of $\phi$ we have an invertible element $U$ of $B(X)$ for which

$$
\phi(A)=U A U^{-1} \quad \text { and } \quad \phi(B)=U B U^{-1} .
$$

Since $B \in F(X)$, we can compute

$$
\begin{aligned}
& T B T^{-1} \phi(A) T B T^{-1}=\phi(B) \phi(A) \phi(B) \\
= & U B U^{-1} U A U^{-1} U B U^{-1}=U B A B U^{-1} .
\end{aligned}
$$

Taking traces in this equality, we get

$$
f\left(T^{-1} \phi(A) T x\right) f(x)=f(A x) f(x) .
$$

This implies that for any $x \in X, f \in X^{\prime}$ with $f(x) \neq 0$ we have

$$
f\left(T^{-1} \phi(A) T x\right)=f(A x) .
$$

Moreover, if $x \in X, 0 \neq f \in X^{\prime}$ and $f(x)=0$, then we can find a sequence $\left(x_{n}\right)$ in $X$ such that $x_{n} \rightarrow x$ and $f\left(x_{n}\right) \neq 0$ for every $n \in \mathbb{N}$. Hence, we deduce that the equality (12) holds without any assumption on $x$ and $f$. This gives us that

$$
\phi(A)=T A T^{-1} \quad(A \in \mathcal{A}) .
$$

To complete the proof it remains to show that in fact we have $\phi(A)=A$ for every $A \in \mathcal{A}$ (we need this to prove that $\phi$ is surjective). From (9) we learn that 
$\phi\left(x_{n} \otimes f_{n}\right)=x_{n} \otimes f_{n}$ for every $n \in \mathbb{N}$. Due to (11) this readily imples that $T x_{n}=\epsilon_{n} x_{n}$ and $T^{-1^{\prime}} f_{n}=\delta_{n} f_{n}$ hold for some scalars $\epsilon_{n}, \delta_{n}$. Moreover, we have $\epsilon_{n} \delta_{n}=1$ for every $n \in \mathbb{N}$. Obviously, we can suppose that $\epsilon_{1}=\delta_{1}=1$. We have supposed in (8) that

$$
\phi\left(\sum_{n} \mu_{n} x_{n} \otimes f_{n+1}\right)=\sum_{n} \mu_{n} x_{n} \otimes f_{n+1} .
$$

By (13) this yields

$$
\sum_{n} \mu_{n} T x_{n} \otimes T^{-1^{\prime}} f_{n+1}=\sum_{n} \mu_{n} x_{n} \otimes f_{n+1} .
$$

Therefore, we have

$$
\sum_{n} \epsilon_{n} \delta_{n+1} \mu_{n} x_{n} \otimes f_{n+1}=\sum_{n} \mu_{n} x_{n} \otimes f_{n+1} .
$$

Considering the values of the operators on both sides at $x_{2}, x_{3}, \ldots$ one after the other, in turn we get that $\delta_{2}=1, \epsilon_{2}=1, \delta_{3}=1, \epsilon_{3}=1, \ldots$. Therefore, $T$ is the identity on $X$ and we have

$$
\phi(A)=A \quad(A \in \mathcal{A}) .
$$

This completes the proof.

\section{REFERENCES}

1. J.C. Hou, Rank-preserving linear maps on $B(X)$, Sci. China Ser. A 32 (1989), 929-940. MR 92b: 47052

2. J. Lindenstrauss and L. Tzafriri, Classical Banach Spaces I, Springer-Verlag, 1977. MR 58:17766

3. M. Marcus and B.N. Moyls, Transformations on tensor product spaces, Pacific J. Math. 9 (1959), 1215-1221. MR 21:7219

4. L. Molnár, Some characterizations of the automorphisms of $B(H)$ and $C(X)$, Proc. Amer. Math. Soc. 130 (2002), 111-120.

5. M. Omladič and P. Šemrl, Additive mappings preserving operators of rank one, Linear Algebra Appl. 182 (1993), 239-256. MR 94f:47039

6. G.K. Pedersen, Analysis Now, Springer-Verlag, 1989. MR 90f:46001

7. R. S. Pierce, Associative Algebras, Springer-Verlag, 1982. MR 84c:16001

8. P. Šemrl, Isomorphisms of standard operator algebras, Proc. Amer. Math. Soc. 123 (1995), 1851-1855. MR 95g:47066

9. P. Šemrl, Local automorphisms and derivations on $B(H)$, Proc. Amer. Math. Soc. 125 (1997), 2677-2680. MR 98e:46082

Institute of Mathematics and Informatics, University of Debrecen, 4010 Debrecen, P.O. Box 12, Hungary

E-mail address: molnarl@math.klte.hu 